\title{
Netzwerken für den Erfolg
}

\author{
INKLUSION Die Schwerbehindertenvertretung (SBV) ist quasi \\ "naturwüchsig" die betriebliche Interessenvertretung, die permanent \\ sehr vielschichtig kooperiert, um erfolgreich zu sein. Ein Praxisprojekt \\ der Uni Köln hat das systematisch untersucht - mit dem Koopera- \\ tionspartner IG BCE.
}

VON MATHILDE NIEHAUS UND MARIE SOPHIA HEIDE

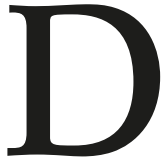
ie SBV ermittelt mit internen und externen Partnerinnen und Partnern berufliche Teilhabemöglichkeiten für behinderte sowie (chronisch) kranke Beschäftigte und stützt sich dabei vor allem auf die im Sozialgesetzbuch IX (SGB IX) rechtlich abgesicherten Kooperationsbeziehungen. Und die SBV eruiert (externe) Leistungsträger für mögliche Teilhabeleistungen - von der medizinischen Reha, über Umschulungen, Einsatzmöglichkeiten und Arbeitszeitmodelle, technische Hilfen und Arbeitsplatzausstattung, Lohnzuschüsse für Arbeitgeber oder Arbeitsassistenz. Die nicht vollständige Liste ist ein Indiz für die große Zahl möglicher Netzwerk-Partner und zuständiger Stellen.

Damit nimmt die Schwerbehindertenvertretung im Hinblick auf die Schaffung einer inklusiven Arbeitswelt und die Ausgestaltung der betrieblichen Prävention und beruflichen Rehabilitation eine besondere und zentrale Rolle ein.

In ihrer Funktion als gewählte Interessenvertretung vertritt sie im gewichtigen $\mathrm{Ma}$ die Rechte der schwerbehinderten und ihnen gleichgestellten Kollegen und Kolleginnen und versucht die Bedarfe zu ermitteln, die für eine bessere betriebliche Inklusion und Teilhabe nötig sind. Um all diesen Aufgaben gerecht zu werden und die Interessenlagen der Zielgruppe gut vertreten und möglichst durchsetzen zu können, sind Netzwerke und Kooperationen das $» \mathrm{~A}$ und $\mathrm{O} \ll$.

\section{Aktiv, wenn Beschäftigte in Not sind}

Im betrieblichen Kontext wenden sich insbesondere von Behinderung bedrohte, chronisch kranke und schwerbehinderte Mitarbeiterinnen und Mitarbeiter an die SBV. In ihrer Rolle als gewählte Interessenvertretung übt die SBV ein betriebliches Ehrenamt aus und übernimmt - neben der Beratung und Unterstützung - vielfältige weitere Aufgaben für den Erhalt der Beschäftigungsfähigkeit.

Die Anliegen der von Behinderung bedrohten und schwerbehinderten Beschäftigten stehen im Vordergrund der Arbeit. Durch persönliche Beratung und unterstützende Aktivitäten arbeitet die Interessenvertretung in einer vertrauensvollen Beziehung mit ihrer Zielgruppe zusammen und profitiert von einer großen Belegschaftsnähe (§ 178 Abs. 1 SGB IX). Die Schwerbehindertenvertretung agiert in diesem Zusammenhang häufig als eine Verbindungsperson zu offiziellen Stellen, sodass Beschäftigte schnelle und pragmatische Hilfe erhalten. Im Folgenden wird zwischen SBV als dem Wahlamt und der SBV als Vertrauensperson unterschieden: also der Person, die das Amt innehat und individuell gestaltet.

\section{Betriebliche Kooperationsbeziehungen}

Die Vertrauenspersonen sind wichtige Ansprechpartner/innen für betriebliche Akteure und Akteurinnen, wie

- für die/den Inklusionsbeauftragte/n des Ar-

\section{DARUM GEHT ES}

1. Die SBV braucht stabile Netzwerke, um innerbetrieblich wie ein Lotse für Inklusion und Prävention wirken zu können.

2. Auch außerbetrieblich sind gute Kontakte nötig, damit die SBV dafür nötige Hilfen und Teilhabeleistungen akquirieren kann.

3. Ein Projekt hat ermittelt, welche Partner und Erfolgsfaktoren zum erfolgreichen Netzwerken gehören. 


\section{LESETIPPS}

$\downarrow$ Bundesarbeitsgemeinschaft der Integrationsämter und Hauptfürsorgestellen (BIH, 2019): Behinderung \& Beruf. www.integrationsaemter. de

D BIH (2018) (2018): ZB Spezial. Die Schwerbehindertenvertretung. www. integrationsaemter.de/ files/11/ABC_Fachlexikon_2018_barrierefrei.pdf

$\triangleright$ L. Hodek, A. Sinn-Behrendt, G. Baumann, R. Bruder, M. Kugler, M. Niehaus (2015): PINA - Gesund und qualifiziert älter werden in der Automobilindustrie. Partizipation und Inklusion von Anfang an. www.pina-projekt.de (Leitfaden gute Kooperation).

$\triangleright$ Institut der deutschen Wirtschaft Köln e.V. (2018): REHADAT-Portal. Schwerbehindertenvertretung (SBV). www. rehadat-recht.de/de/ schwerbehindertenvertretung-sbv/index.html. beitgebers ( $\$ 181$ SGB IX)

für den Betriebs- /Personalrat (§ 178 Abs. 4 SGB IX)

sowie für den Arbeitgeber selbst ( $\$ 178$ Abs. 2 SGB IX).

Neben der gesetzlichen Verankerung der $\mathrm{Zu}$ sammenarbeit von SBV, Arbeitgeber, Inklusionsbeauftragtem, Betriebs- oder Personalrat nach $\S 182$ Sozialgesetzbuch IX (SGB IX) ist zudem verbindlich geregelt: Die SBV hat ein Teilnahmerecht an den Sitzungen des Betriebs- oder Personalrats und seinen Ausschüssen (§ 178 Abs. 4 SGB IX). Sie nimmt beratend teil und kann beantragen, dass ihre Themen auf der Tagesordnung berücksichtigt werden (Teilhabe, inklusive Ausbildung, Gesundheitsprävention etc.).

Bei allen Themen, die schwerbehinderte Bewerberinnen und Bewerber oder Mitarbeiter/innen betreffen, muss der Arbeitgeber die SBV » unverzüglich und umfassend « informieren und zur Teilnahme an Gesprächen einladen (§ 178 Abs. 2 SGB IX). Nur so kann sie ihre Aufgaben und die Interessenvertretungsarbeit rechtskonform wahrnehmen. Die Kündigung eines schwerbehinderten Menschen ohne ihre Beteiligung und Anhörung ist unwirksam. ${ }^{1}$

Gemeinsam mit dem Betriebs- oder Personalrat und dem Inklusionsbeauftragten des Arbeitgebers agiert die SBV in einem betrieblichen Integrationsteam ( $\S 176,178$ und 181 SGB IX). Das betriebliche Integrationsteam wirkt bei der Erarbeitung und beim Abschluss einer Inklusionsvereinbarung mit, es behandelt Fragestellungen der Prävention und des Betrieblichen Eingliederungsmanagements
(BEM, § 167 Abs. 2 SGB IX $\left.{ }^{2}\right)^{3}$ Den Arbeitgeber berät die Vertrauensperson in individuellen und generellen Fragestellungen zur beruflichen Teilhabe schwerbehinderter und ihnen gleichgestellter Menschen und überprüft die Einhaltung gesetzlicher Pflichten $(\S 178$ Abs. 1 SGB IX).

\section{Die SBV als Gesundheitslotse}

Im Sozialrecht gilt der Grundsatz »Prävention vor Rehabilitation, Rehabilitation vor Rente«. Prävention nach $\S 3$ SGB IX ist ebenso wie die Rehabilitation darauf gerichtet, die Beschäftigungsfähigkeit zu erhalten sowie den vorzeitigen Eintritt von Pflegebedürftigkeit zu vermeiden und Zugängen in die Eingliederungshilfe frühzeitig entgegenzuwirken. Prävention nach $\S 3$ SGB IX bedeutet insbesondere auch, betrieblich tätig zu werden und Unterstützung zu leisten, bevor Rehabilitationsbedarf oder der Verlust des Arbeitsverhältnisses droht (»Vorrang von Prävention«). Insofern ist Prävention am Arbeitsplatz neben der Rehabilitation ein eigenständiges Handlungsfeld der SBV.

Die frühzeitige und umfassende Identifizierung von Bedarfen und die Einleitung geeigneter präventiver und rehabilitativer Maßnahmen im betrieblichen Alltag ist eine Aufgabe, die der SBV unweigerlich zufällt; sie nimmt damit im Rahmen der betrieblichen Zusammenarbeit mit anderen Akteurinnen und Akteuren die Funktion einer Art "Gesundheitslotse « ein. Betriebliche Partner sind u. a. - neben dem Arbeitgeber, dem Inklusionsbeauftragten, dem Betriebs-/Personalrat: die Personalabteilung, Fachkräfte für Arbeitssicherheit, Betriebsmedizin, Sozialberatung etc. ${ }^{4}$

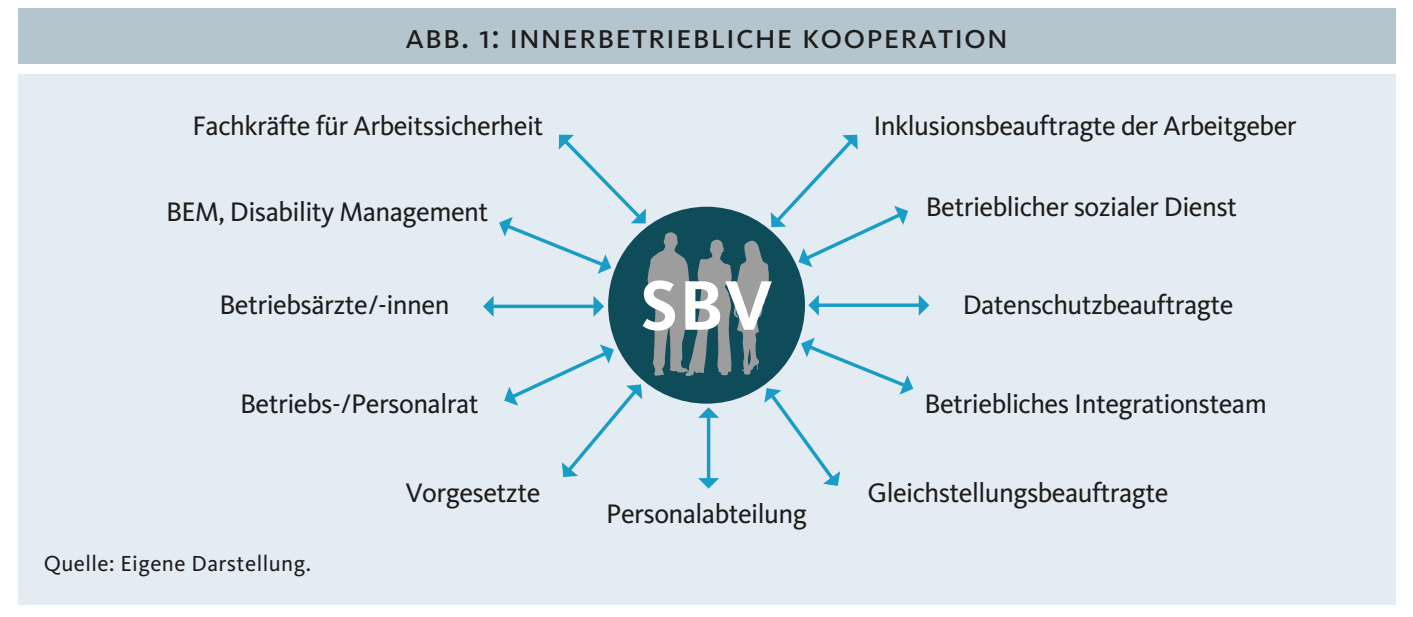

1 Neu im SGB IX nach dem Bundesteilhabegesetz (BTHG) 2016.

\footnotetext{
Vgl. dazu auch »Gute Arbeit « 5/2019 (S. 36 ff.): BEM - Eingliederung ohne Stolpersteine.

Bundesarbeitsgemeinschaft der Integrationsämter und Hauptfürsorgestellen (BIH) (2018): Die Schwerbehindertenvertretung, Wiesbaden.

4 Unter Wahrung des Datenschutzes: Es geht um Arbeitsplatzgestaltung, nicht um Diagnosen ( $\$ 178$ Abs. 3 SGB IX).
} 
ABB. 2: AUSSERBETRIEBLICHE KOOPERATION

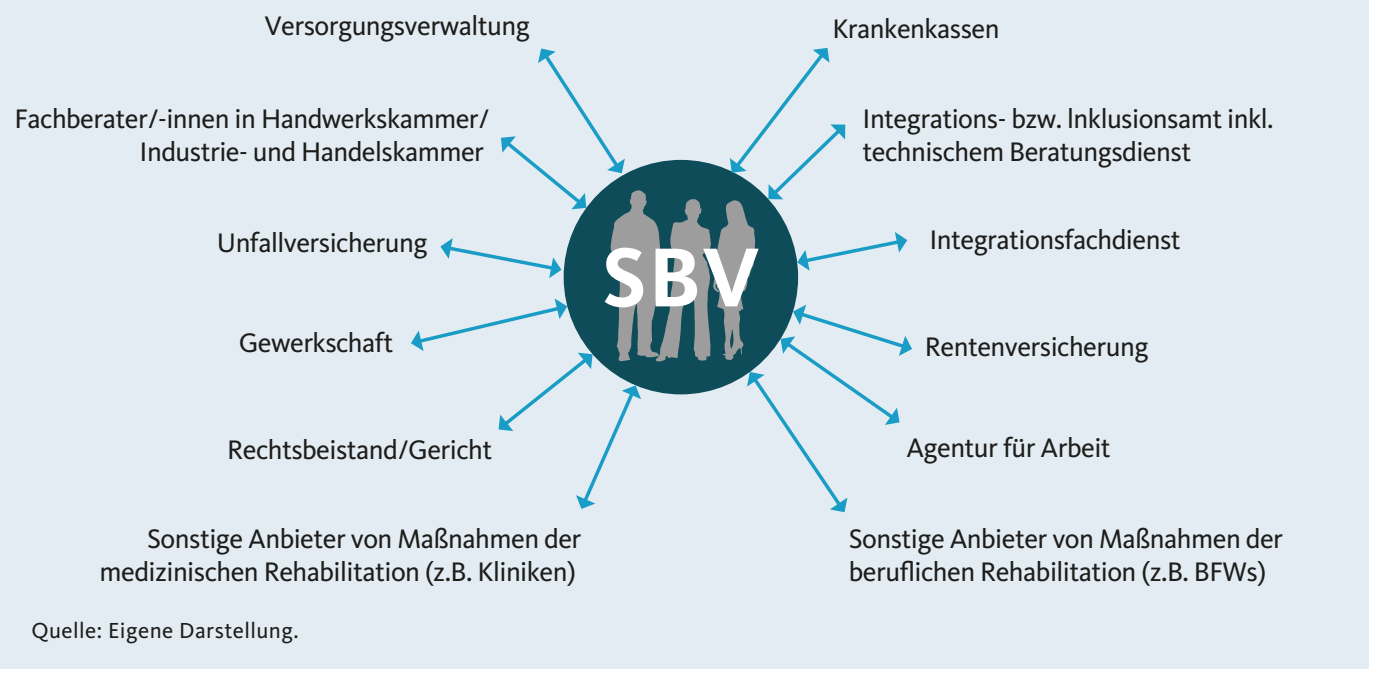

\section{SBV-Netzwerke als effektive Unterstützung}

Damit die Vertrauenspersonen und ihre Stellvertreter/innen diese Aufgaben als Lotsen und innerbetriebliche Funktionsträger/innen für berufliche Rehabilitation bzw. als Koordinator(inn)en zwischen Betrieb einerseits und Rehabilitationsträgern, Integrations- bzw. Inklusionsämtern und Leistungserbringern andererseits erfüllen können, benötigen sie Freiräume bei der Gestaltung ihrer Arbeit.

Die Vernetzung mit anderen Akteuren und Akteurinnen ist eine bedeutende Ressource für die Arbeit der SBV. Die Zusammenarbeit mit Fachleuten und den Vertreter(inne)n externer Stellen (Leistungsträger) hilft beim Ziel Inklusion, bei der Eingliederung - mit praktischen Hilfen und Unterstützungsleistungen.

Es kann aber auch vorkommen, dass in der Zusammenarbeit mit einzelnen Akteuren Konflikte auftreten. Für das Auflösen möglicher Streitigkeiten und Konflikte gibt es keine allgemeingültigen Lösungen. Hilfreich sind gezielte Weiterbildungen für die Entwicklung persönlicher und sozialer Kompetenzen für das Amt, die zum Beispiel die Gewerkschaften, gewerkschaftliche Bildungsträger und die Integrationsämter und anbieten.

\section{Wie sehen SBV-Netzwerke aus?}

Die Netzwerke der Vertrauenspersonen sind je nach Betrieb und Thema unterschiedlich gestaltet, zum Beispiel auch hinsichtlich der Zusammensetzung und der Intensität der $\mathrm{Zu}$ sammenarbeit.

Um die Netzwerkarbeit verbessern $\mathrm{zu}$ können, ist ein erster wichtiger Schritt die Bestandsaufnahme: Wie sieht mein bisheriges Netzwerk aus? Welche Partner/innen und Unterstützer/innen habe ich? Was läuft rund, wo treten regelmäßig Konflikte auf? Die Beantwortung folgender Fragen kann bei der Auseinandersetzung mit dem eigenen Netzwerk hilfreich sein:

- Welche Akteure und Akteurinnen sind für meine Arbeit als Schwerbehindertenvertretung hilfreich?

- Welche Akteure und Akteurinnen nehmen eine unterstützende Rolle ein?

- In welchen Beziehungen treten Konflikte auf?

- Stehen die Akteure und Akteurinnen untereinander in Kontakt?

- Sind alle wichtigen Partner/Stellen/Institutionen in meinem Netzwerk vertreten?

In der Online-Befragung der Universität Köln antworteten 1552 Vertrauenspersonen auf die Fragen, mit wem sie zusammenarbeiten und wie viel Zeit sie mit wem und welchen Tätigkeiten verbringen. Auf dieser Grundlage zeigt sich, dass die Interessenvertretungen in ihrem Amt als Schwerbehindertenvertretung im Durchschnitt 31\% ihrer Zeit für Kooperationen mit inner- und außerbetrieblichen Akteuren und Akteurinnen verwenden.

\section{Stemmt das Neue mit Bravour}

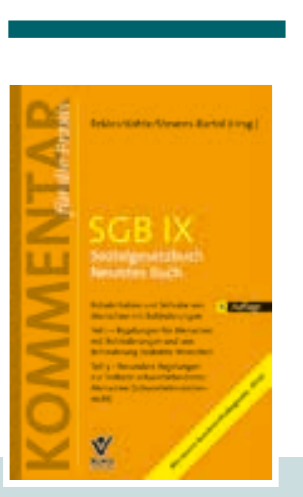

Feldes / Kothe / Stevens-Bartol (Hrsg.) SGB IX

Kommentar für die Praxis

4., überarbeitete $u$. aktualisierte Auflage 2018. 1.565 Seiten, gebunden

$€ 109$,-

ISBN 978-3-7663-6719-8

www.bund-verlag.de/6719

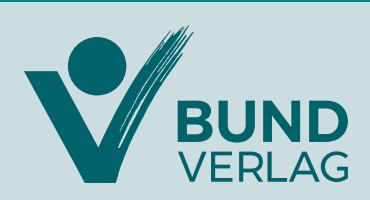

kontakt@bund-verlag.de Info-Telefon: 069/7950 10-20 
Der Lehrstuhl für Arbeit und Berufliche Rehabilitation der Universität zu Köln (Prof. Dr. Mathilde Niehaus) hat in Zusammenarbeit mit der Industriegewerkschaft Bergbau, Chemie, Energie im Rahmen des von der Hans-Böckler-Stiftung geförderten Projekts »Schwerbehindertenvertretungen: Allianzpartner in Netzwerken« die Rolle der Schwerbehindertenvertretung in Deutschland untersucht.

Bundesweit wurden Ende 2017 insgesamt 1552 Vertrauenspersonen schriftlich per Online-Befragung zu ihrem Amt als Schwerbehindertenvertretung (SBV) befragt, darauf aufbau- end wurden für eine tiefergehende Analyse leitfadengestützte Interviews mit einzelnen Vertrauenspersonen geführt. Zudem fanden Fokusgruppendiskussionen mit der SBV und ihren inner- und außerbetrieblichen Kooperationspartnern statt. Im Anschluss wurde eine Zukunftswerkstatt mit Akteuren und Akteurinnen aus der betrieblichen Praxis durchgeführt. Projektergebnisse nachlesen: M. Niehaus, M.S. Heide, A. Glatz (2019): Von Anfang an zusammen. Handlungsleitfaden für Schwerbehindertenvertretungen in Netzwerken. Düsseldorf. Link zur PDF-Datei: www.boeckler. $\mathrm{de} / \mathrm{pdf} /$ p_mbf_praxis_2019_22.pdf
Die durchschnittliche Zeit von 31\% setzt sich so zusammen:

- 23\% für die Kooperationen mit innerbetrieblichen Akteuren

8\% für die Kooperationen mit außerbetrieblichen Akteuren.
Wie vielseitig die Kooperationsbeziehungen der SBV sind, zeigen die Abbildungen 1 und 2: Abb. 1 zeigt die innerbetriebliche Partner/ innen, die Abb. 2 stellt mögliche außerbetriebliche Akteure vor, mit denen die Schwerbehindertenvertretungen regelmäßig zu tun haben.

ABB. 3: TIPPS FÜR GELINGENDE NETZWERKARBEIT DER SCHWERBEHINDERTENVERTRETUNGEN

1. Vertrauen und
persönliche Kommunikation
Ein vertrauensvoller Umgang
miteinander und persönliche
Kommunikation ist die Basis
für eine gute Zusammenarbeit.

4. Klärung der Aufgaben im Netzwerk durch klare Rahmung

Der Rahmen der Zusammenarbeit sollte im Netzwerk klar abgesteckt und die jeweiligen Aufgaben sollten definiert sein.

\section{Kontaktdaten möglicher} Ansprechpartner/innen

Für die Zusammenarbeit mit verschiedenen Akteuren und Akteurinnen sollte die Kommunikation strukturiert verlaufen und die Kontaktaufnahme leicht sein.

\section{Verfügbarkeit von Ressourcen}

Für die Zusammenarbeit mit anderen Akteuren und Akteurinnen braucht es zeitliche Ressourcen.

\section{Soziale und fachliche Kompe-} tenzen der Netzwerkakteure und-akteurinnen

Soziale und fachliche Kompetenzen sollten aufseiten der Schwerbehindertenvertretung und aufseiten des Akteurs bzw. der Akteurin vorhanden sein.

\section{Transparenz}

Es ist hilfreich für die Zusammenarbeit mit anderen Akteuren und Akteurinnen, wenn die Abläufe und Ziele der Arbeit einer Schwerbehindertenvertretung bekannt sind.

\section{Aktives Netzwerk-} management

Es ist wichtig, dass es eine Person in der Zusammenarbeit gibt, die die Rolle des Promoters oder der Promoterin einnimmt. Diese Person koordiniert das Netzwerk und treibt es voran.

\section{Regelmäßigkeit und} Stabilität des Netzwerks

Für die Zusammenarbeit mit anderen Akteuren und Akteurinnen ist es wichtig, dass es einen regelmäßigen Austausch innerhalb des Netzwerks gibt.

\section{9. Öffentlichkeitsarbeit}

Es sollte eine gemeinsame Öffentlichkeitsarbeit stattfinden, damit das Netzwerk von möglichst vielen Kollegen und Kolleginnen gesehen wird.

Quelle: Eigene Darstellung. 


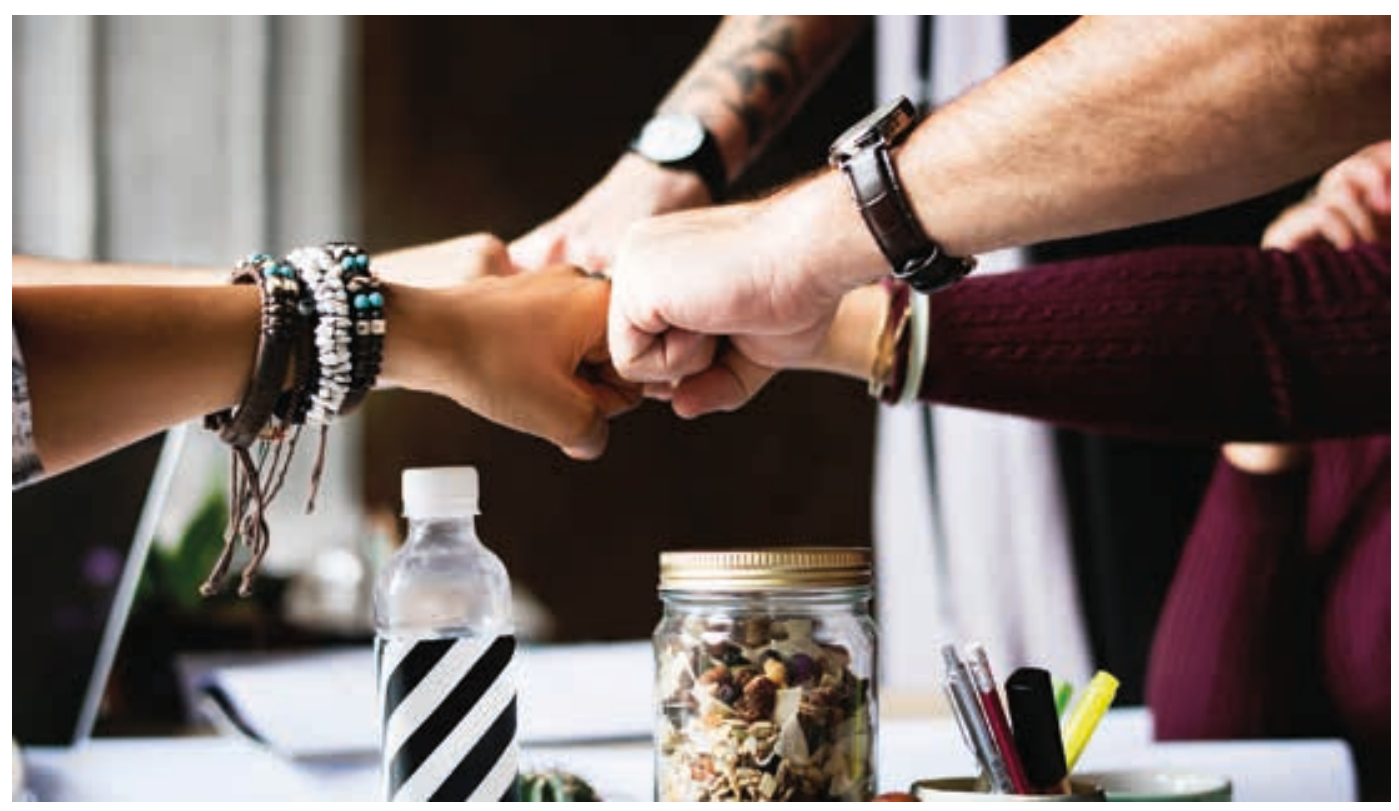

\section{Tipps für erfolgreiche Netzwerkarbeit}

Das Netzwerk der Schwerbehindertenvertretung hat im Idealfall das gemeinsame Ziel, die Beschäftigungsfähigkeit der Kollegen und Kolleginnen zu erhalten, die Gesundheit und die Inklusion in das Arbeitsleben zu fördern.

Wie kann die Zusammenarbeit mit den verschiedenen inner- und außerbetrieblichen Akteuren und Akteurinnen gelingen? Wie kann ein Netzwerk bestmöglich genutzt werden? Wie können Ziele möglichst optimal und gemeinsam von den Partnerinnen und Partnern erreicht werden?

In der Abbildung 3 werden neun konkrete Punkte vorgestellt, die für die Arbeit im Netzwerk hilfreich sein können. Diese Erfolgsfaktoren wurden im Jahr 2012 von Expertinnen und Experten aus dem Bereich der Rehabilitation erarbeitet und von der Bundesarbeitsgemeinschaft für Rehabilitation e.V. (BAR e.V.) zusammengefasst. ${ }^{5}$ Gemeinsam mit Vertrauenspersonen wurden die Punkte diskutiert und für die Netzwerkarbeit im Amt der Schwerbehindertenvertretung angepasst. In der täglichen Praxis sind diese Erfolgsfaktoren nicht isoliert zu sehen, vielmehr wirken sie zusammen und verstärken sich gegenseitig. Sie betreffen verschiedene Ebenen:

die Vertrauensebene der Akteure

die Verfügbarkeit von Ressourcen (insbesondere Zeitbedarf!) für ein aktives Netzwerkmanagement mit Wirkung nach außen

Verabredungen und Routinen über Art und
Häufigkeit des Austausches, die Transparenz über Abläufe und Ziele und die damit verbundene Aufgabenverteilung im Netzwerk.

\section{Fazit}

Eine ausführlichere Darstellung der Netzwerke von Schwerbehindertenvertretungen befinden sich in der Publikation: "Von Anfang an zusammen. Handlungsleitfaden für Schwerbehindertenvertretungen in Netzwerken " (vgl. Angaben im Kasten S. 58). Enthalten sind Beispiele für SBV-Netzwerke (mit den unterschiedlichen Stellen) sowie eine Netzwerkkarte zum Ausfüllen, die bei der Auseinandersetzung mit dem eigenen betrieblichen und außerbetrieblichen Netzwerk hilft.

Darüber hinaus enthält die Handlungshilfe Tipps mit Hinweisen aus der betrieblichen Praxis, die eine gelingende Netzwerkarbeit der Schwerbehindertenvertretung fördern und die Prüfung der betrieblichen Standards ermöglichen. $\triangleleft$

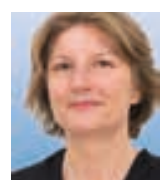

Prof. Dr. Mathilde Niehaus, Professur für Arbeit und Berufliche Rehabilitation an der Universität Köln.

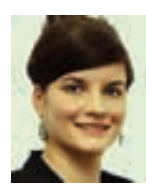

Marie Sophia Heide (M.A.), wiss. Mitarbeiterin am Lehrstuhl für Arbeit und Berufliche Rehabilitation der Universität Köln.

\begin{abstract}
Die SBV ist nicht "everybody's Darling", sondern eine Interessenvertretung. Trotzdem ist Kooperation und Arbeit in Netzwerken ein entscheidender Faktor, um sich durchzusetzen und Wirkung zu erlangen.
\end{abstract}

\section{Fit für die neue SBV-Amtszeit}

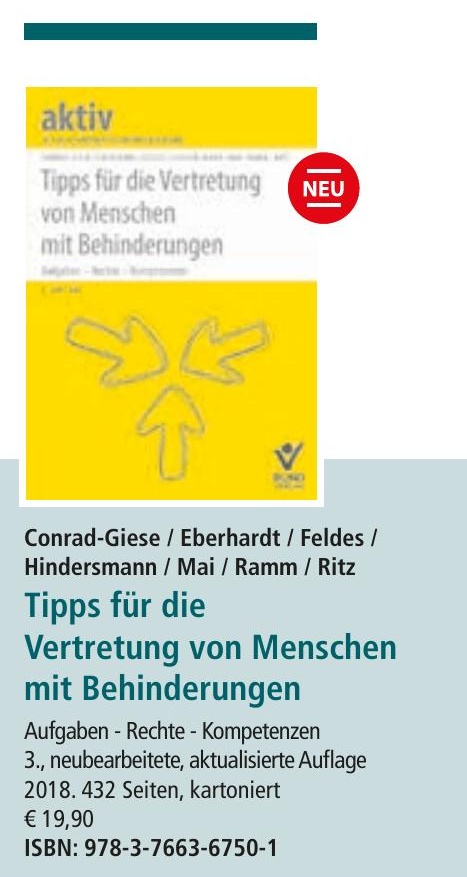

www.bund-verlag.de/6750

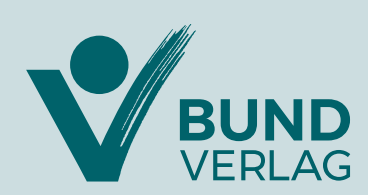

kontakt@bund-verlag.de Info-Telefon: 069/795010-20 